\title{
Efficacy of rufinamide in childhood refractory epilepsy
}

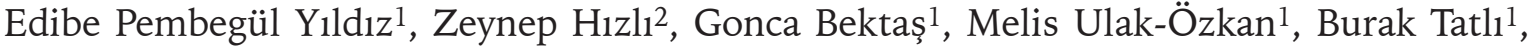 \\ Nur Aydınlı1 ${ }^{1}$, Mine Çalışkan ${ }^{1}$, Meral Özmen ${ }^{1}$ \\ Departments of ${ }^{1}$ Pediatric Neurology and ${ }^{2}$ Pediatrics, Istanbul University Istanbul Faculty of Medicine, Istanbul, Turkey. \\ E-mail: edibepembegul@hotmail.com \\ Received: 16th March 2017, Accepted: 4th July 2017
}

SUMMARY: Yıldız EP, Hızlı Z, Bektaş G, Ulak-Özkan M, Tatlı B, Aydınlı N, Çalışkan M, Özmen M. Efficacy of rufinamide in childhood refractory epilepsy. Turk J Pediatr 2018; 60: 238-243.

Rufinamide has been used as a new antiepileptic drug in the treatment of drug-resistant epilepsy, in recent years. The objective of this study was to evaluate the reliability of rufinamide and its impact on seizure frequency in patients diagnosed with drug-resistant epilepsy, where seizures could not be controlled with 'classical' antiepileptic drugs. We retrospectively reviewed the data of epileptic patients who were followed up between January 2004 and December 2014 in the Pediatric Neurology Department. Patients who were diagnosed with 'drug resistant epilepsy' and treated with rufinamide were evaluated. Decrease in seizure frequency and drug side effects were assessed as parameters. A total of 38 patients (14 girls, 24 boys) with a mean age of 8.5 (range, 3.5-17) years were included in the study. The mean follow-up duration was 25.5 (23-29.5) months, while the mean maximal dose of rufinamide was $32.5(28-42) \mathrm{mg} / \mathrm{kg} / \mathrm{day}$. Response to treatment was assessed by the reduction in frequency of seizures. The decrease was $<50 \%$ (essentially unresponsive to treatment) in 20 patients and $50-99 \%$ in 8 patients. Ten patients $(26.3 \%)$ remained seizure-free. The response rate for tonic seizures was $50 \%$. In drop/attacks seizures, this ratio was found as $73 \%$, which was quite high. Patients with myoclonic and tonic-clonic seizures did not significantly benefit from rufinamide. The rate of patients with Lennox-Gestaut syndrome (LGS) who responded very well (reduction in seizure frequency $>50 \%$ ) was $55.5 \%$. In the LGS group, patients with drop/attacks showed the best response to treatment. Rufinamide was not effective in two patients diagnosed with Dravet syndrome. Rufinamide can be safely used in pediatric patients who use multiple antiepileptic drugs and are unresponsive to the treatment. It was seen to be effective especially in patients diagnosed with LGS and drop/attacks types of seizures.

Key words: rufinamide, childhood, drug resistant epilepsy, pediatrics.

Although remission is reported in $60-70 \%$ of pediatric epilepsy patients with appropriate and adequate antiepileptic therapy ${ }^{1}$, treatment is quite difficult in this group of patients. In recent years, rufinamide has drawn attention as an effective adjunctive therapy, promising to reduce the frequency of seizures in this group of patients2. Recently, many studies have been published about the use of rufinamide in the treatment of intractable epilepsy of childhood. Rufinamide was approved in both Europe (2007) and USA (2008) to be used in patients aged four years and older, as an adjunctive treatment agent in seizures with Lennox-Gastaut Syndrome (LGS).2,3 Moreover, the activity and tolerability of rufinamide in patients aged under four years have been shown in several publications and larger studies performed in both adults and early childhood. ${ }^{4-6}$

Rufinamide is a triazole derivative agent, structurally distinct from the other antiepileptic drugs. It is believed to inactivate voltage-gated sodium channels, resulting in the stabilization of membranes and, by this way, decreasing neuron firing and consequently preventing the development of seizures. Rufinamide is 
well-absorbed with a single oral administration with more than $85 \%$ bioavailability has been reported. $4,5,7$ It is widely metabolized in the body, and cytochrome P450 isozymes are apparently not involved in this process. ${ }^{8}$

The objective of this study was to evaluate the reliability of rufinamide and its impact on seizure frequency in patients diagnosed with drug-resistant epilepsy, where seizures cannot be controlled with 'classical' antiepileptic drugs.

\section{Material and Methods}

This retrospective study was approved by the Institutional Review Board of our university hospital and performed in accordance with the Declaration of Helsinki.

\section{Case identification}

We retrospectively reviewed the data of epileptic patients who were followed up between January 2004 and December 2014 in the Pediatric Neurology Department. Patients who were diagnosed with 'drug resistant epilepsy' and treated with rufinamide were evaluated. Patients' data were recorded including age, gender, age of seizure onset, seizure type, etiology of seizure, electroclinic syndromes, previously used antiepileptic treatments, electroencephalogram (EEG) findings, initiation times, doses and maximal doses of rufinamide, duration of treatment, and adverse effects. Patients diagnosed with neurodegenerative diseases were excluded.

Rufinamide was administered at a dose of 10 $\mathrm{mg} / \mathrm{kg} /$ day, followed by a week of titration with increments of $10 \mathrm{mg} / \mathrm{kg}$ up to the final dose. The titration phase was prolonged on the basis of the clinical condition, in the case that adverse effects developed. Decrease in seizure frequency and drug side effects were assessed as parameters.

\section{Data analysis}

Responses to treatment was evaluated by monitoring the reduction in seizure frequency compared with the initial frequencies, and grouped as follows: (1) group 1: completely controlled seizures (100\% response), (2) group 2: $50-99 \%$ reduction in frequency of seizures, (3) group 3: less than $49 \%$ reduction or no change in frequency of seizures (no response to treatment). Groups 1 and 2 were considered as rufinamide responsive.

\section{Definitions}

Drug-resistant epilepsy (DRE) is defined as failure of two or more anti-epileptic drugs with seizure frequency of more than one every six months in the year immediately before final follow-up. ${ }^{9}$

For each patient, epilepsy was classified according to the new ILAE Commission on Classification and Terminology 2011-2013 Report. ${ }^{10}$ Factors considered in the classification included seizure type(s) based on descriptive semiologies from the medical record, EEG and neuroimaging findings, and for some specific syndromes, age at onset. For each case, epilepsy was classified based on mode of onset at presentation (generalized, focal, or unknown) and etiology (genetic, structural, metabolic, infection or unknown).

\section{Statistical analysis}

All statistical analyses were applied using SPSS Statistics for Windows version 16.0 (IBM Corp, Armonk, NY, USA). Data are presented as median, minimum, maximum, frequency, and percentage.

\section{Results}

A total of 38 patients (14 girls, 24 boys) with a mean age of 8.5 (range, 3.5-17) years were retrospectively analyzed. The patients were followed-up with the diagnosis of DRE and rufinamide was added to their treatment regimen. The mean follow-up duration was 25.5 (23-29.5) months, while the mean maximal dose of rufinamide was $32.5(28-42) \mathrm{mg} / \mathrm{kg} /$ day.

Etiology of seizures was evaluated in the patients based on medical history, physical examination, laboratory investigations, imaging techniques and metabolic screening tests. The frequency of etiologic factors is shown in Table I.

Responses of our patients to rufinamide by etiology of epilepsy are given in Table II. Whereas number of seizures did not chane in patients with infection induced epilepsy, the average rate of response to drug was found as $50 \%$ in the patients in all other etiology groups. 
Table I. Etiology of Epilepsy.

\begin{tabular}{ll}
\hline Etiology of epilepsy & No of patient \\
\hline Genetics & 7 \\
Structural* $^{*}$ & 11 \\
Metabolic disease & 2 \\
Infection & 3 \\
Unknown & 15 \\
\hline
\end{tabular}

*Hypoxic-ischemic encephalopathy 6, Cortical dysplasia 1 , Neurocutaneus disease 2, stroke 2

Response to treatment was assessed by the reduction in frequency of seizures. The decrease was $<50 \%$ (essentially unresponsive to treatment) in $20(52.6 \%)$ patients and $50-99 \%$ in $8(21 \%)$ patients. Ten patients $(26.3 \%)$ remained seizure-free ( $100 \%$ response). (Fig. 1). One patient with unknown etiology, who had myoclonic seizures, showed a complete response on the seventh day of the treatment, but seizures resumed in the third week. Another patient who had intractable seizures due to propionic acidemia and a previous history of stroke, responded to treatment at the beginning, but seizures began again at the end of the third month. In one patient diagnosed with LKS, the seizures restarted at the end of the first month. These three patients initially exhibited full responses to treatment, but seizures resumed at the third week, first week and third month during follow-up period; respectively. These patients did not benefit from dose titration and thus they were included in the unresponsive patient group. One patient with unknown etiology was followed-up as seizure free over 8 months. However, seizures recurred after the 8 th month despite no change was made in the treatment and regular use of drugs. Seizures continued in the patient who was on $40 \mathrm{mg} / \mathrm{kg} / \mathrm{g}$ rufinamide and therefore, this patient was also considered in Group 2.

Fourteen of our patients had focal $(36 \%)$ and 24 generalized (64\%) seizures. Four of the $14(28.4 \%)$ patients with focal epilepsy (symptomatic/idiopathic) and 14 of $24(58 \%)$ patients with generalized epilepsy (seizure-free in 8 patients) responded well to rufinamide.

The response rate for tonic seizures was $50 \%$ $(2 / 4)$. In drop/attacks seizures, this ratio was found as $73 \%(11 / 15 ; 8$ patients showed complete responses), which was quite high. Patients with myoclonic (0/2) and tonic-clonic $(1 / 3)$ seizures did not significantly benefit from rufinamide.

Out of 38 patients, 18 were diagnosed with LGS, 3 with Landau-Klefner syndrome (LKS), and 2 with Dravet syndrome.

The rate of patients with LGS who responded very well to rufinamide, which means $>50 \%$ reduction in seizure frequency $>50 \%$, was found as $55.5 \%$ ( $7 / 10$ patients presented full responses). In the LGS group, patients

Table II. The Distribution of Seizure Type and Etiologies Among Groups.

\begin{tabular}{lccc}
\hline Variable & $\begin{array}{c}\text { Group } 1 \\
\mathrm{n}(\%)\end{array}$ & $\begin{array}{c}\text { Group } 2 \\
\mathrm{n}(\%)\end{array}$ & $\begin{array}{c}\text { Group } 3 \\
\mathrm{n}(\%)\end{array}$ \\
\hline Seizure type & & & \\
$\quad$ Generalized & $8(33)$ & $6(25)$ & $10(41)$ \\
$\quad$ Tonic & 0 & $2(33)$ & $2(20)$ \\
$\quad$ Drop/attacks & $8(100)$ & $3(50)$ & $4(40)$ \\
$\quad$ Myoclonic & 0 & 0 & $2(20)$ \\
$\quad$ Tonic-clonic & 0 & $1(16)$ & $2(20)$ \\
Focal & $2(14)$ & $2(16)$ & $10(71)$ \\
& & & \\
Etiology & $2(29)$ & $1(14)$ & $4(57)$ \\
$\quad$ Genetics & $3(27)$ & $3(27)$ & $5(45)$ \\
Structural* & 0 & $1(50)$ & $1(50)$ \\
Metabolic disease & 0 & 0 & $3(100)$ \\
Infection & $5(33)$ & $3(20)$ & $7(46)$ \\
$\quad$ Unknown & & & \\
\hline
\end{tabular}

*Hypoxic-ischemic encephalopathy 6 , cortical dysplasia 1 , neurocutaneus disease 2, stroke 2 
with drop/attacks showed the best response to treatment (5/7 patients). Rufinamide was not effective in two patients diagnosed with Dravet syndrome.

Considering the clinical and laboratory findings, 7 patients developed side effects including drowsiness, nausea and vomiting. One patient with neurofibromatosis type 1 .and one patient in the LGS group, in whom the etiology could not be determined, showed increased frequency of seizures.

Informed consent was obtained from all individual participants included in the study.

\section{Discussion}

Many studies on small numbers of patients have shown that rufinamide is an effective adjunctive medication in the treatment of drug resistant epilepsy of childhood. In this study, activity and tolerability of rufinamide in pediatric patients were evaluated by seizure type and epileptic syndromes. In total, frequency of seizures decreased by more than $50 \%$ with treatment of 25.5 months on average in $18(47.3 \%)$ patients. A study including 45 children and 15 adults with refractory epilepsy, evaluated the effect of rufinamide added to the treatment regimen and showed a decrease in number of seizures by more than $50 \%$ in 28 patients ${ }^{5}$. Grosso et al. ${ }^{6}$ reported that addition of rufinamide to the treatment caused more than a $50 \%$ reduction in frequency of seizures after a mean follow-up duration of 12.2 months in 11 of $40(27.5 \%)$ patients under four years old with drug resistant epilepsy. We believe that, these differences in outcomes are due

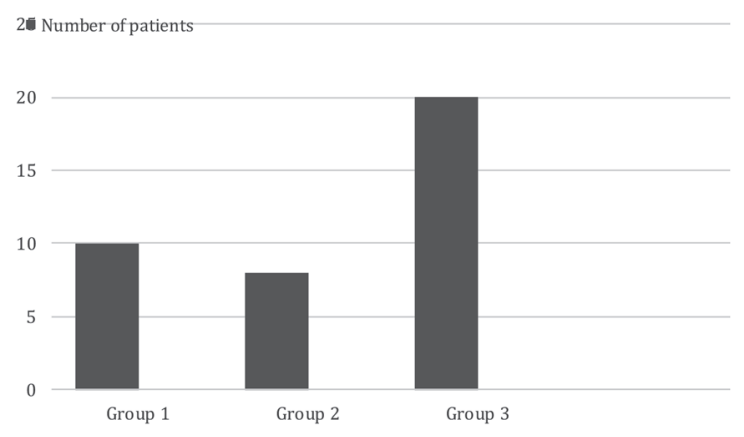

Fig. 1. Changes in seizure frequency with rufinamide therapy.) group 1: $100 \%$ response, group 2: $50-99 \%$ reduction in seizure frequency, group 3: less than $49 \%$ reduction or no change in seizure frequency (no response to treatment). to follow-up period, age group, number of epileptic syndromes, and etiology.

Studies have been reported in the literature evaluating the effectiveness of rufinamide in different types of seizures. In our study, the use of rufinamide was found to be efficient in 50\% of patients with tonic seizures, consistent with the literature. ${ }^{3,6}$ However, rufinamide was not effective in patients with myoclonic seizures. Unlike studies demonstrating effectiveness of rufinamide in tonic-clonic seizures, in our study it was not effective in patients with this type of seizure. ${ }^{11}$ We achieved the best results in patients with drop/attacks seizures. In this group, the number of seizures decreased by more than $50 \%$ in 11 of $15(73 \%)$ patients, while $8(53 \%)$ of the patients were considered seizure-free. Grosso et al. ${ }^{6}$ reported that rufinamide was effective in $42 \%$ of children who had drop/attacks. Coppola et al. ${ }^{3}$ reported more than $50 \%$ reduction of seizures in 38 patients aged 4-43 years diagnosed with epileptic encephalopathies other than LGS and in 7 of $11(63 \%)$ patients who had drop/attacks. Similar rates were recorded in different studies, that have evaluated the effect of rufinamide on LGS patients with drop/attacks. ${ }^{3,6,12}$ In our study, this rate was slightly higher than other studies and consistent with the rate reported by Coppola et al. ${ }^{3}$ We attributed this difference to non-equal distribution of our patients among the types of seizures.

Regarding the efficacy of rufinamide in epileptic syndromes, in the present study adjunctive rufinamide treatment resulted in $>50 \%$ reduction in frequency of seizures in 10 of $18(55.5 \%)$ patients diagnosed with LGS. Seven of these patients were recorded as seizure-free at their last follow-up. Previous studies have shown that rufinamide is highly effective in seizures caused by LGS. In a doubleblinded randomized study involving 74 patients, the treatment response was $50 \% .{ }^{13}$ Other prospective and retrospective studies support similar antiepileptic efficacy of rufinamide in seizures associated with LGS. In individualbased studies, the proportions of patients responding to treatment of rufinamide were reported between $30 \%$ and $60 \%$ based on a $>50 \%$ reduction in frequency of seizures. ${ }^{14-16}$ In our study, rufinamide was not effective in two patients diagnosed with Dravet syndrome. 
Müller et al. ${ }^{17}$ also observed that rufinamide was not highly effective in Dravet syndrome and only $20 \%$ of patients at 6 months and $5 \%$ of patients at 34 months were responsive to treatment. None of four patients in the study by Coppola et al. ${ }^{3}$ benefited from rufinamide.

In our study, one of three patients with LKS was seizure-free in the sixth month of treatment, and another was seizure-free up to three months, at which point, his seizures began again. In our last mentioned patient, adding rufinamide to the treatment regimen showed no change in frequency of seizures.

In a study evaluating 70 patients aged 3-21 years, the response rate in focal seizures was found as $38.5 \%$ in the twelfth month of treatment. ${ }^{18}$ A lower response rate in focal seizures was observed in our study $(25 \%)$, but it was similar to that of Grosso et al. ${ }^{6}$ (30\%).

The most frequent side effects of rufinamide treatment are drowsiness, nausea and vomiting. In our study, nausea and vomiting were observed by $19 \%$, consistent with the literature ${ }^{11}$, but it was not a severe enough clinical condition to cause discontinuation of medication. We did not observe any significant serious side effects. In our patients, discontinuation of medication was due to lack of effectiveness rather than side effects. Only one patient with LGS (unknown etiology) and one patient with neurofibromatosis showed significant increased frequency of seizures with the addition of rufinamide.

Two patients who had been diagnosed with methylmalonic acidemia (MMA) and propionic acidemia (PPA) showed continuation of seizures despite taking at least three antiepileptic drugs. Rufinamide treatment was initiated following hospitalization of these patients. They were followed up with daily clinical status, laboratory values, and electrocardiogram (PR and QT interval was evaluated), and the doses were increased gradually. The patient with MMA showed $>50 \%$ reduction in frequency of seizures. The PPA patient was recorded as seizure-free for one month, but this was followed by repeated seizures that did not benefit from increasing the dose. In different studies, rufinamide reduced tonic seizures in two patients with MMA; however, the results of these limited studies should be approached with caution. ${ }^{18,19}$
In conclusion, the efficacy of rufinamide in different epileptic syndromes and seizure types was assessed in this study. The drug was especially effective against drop/attacks in LGS. However, it was not effective in Dravet syndrome. Overall, rufinamide is a welltolerated anticonvulsant drug, and is likely a good option in children who suffer from drug resistant epilepsy.

\section{REFERENCES}

1. Kwan P, Brodie MJ. Early identification of refractory epilepsy. N Engl J Med 2000; 342: 314-319.

2. Vendrame M, Loddenkemper T, Gooty VD, et al. Experience with rufinamide in a pediatric population: A single center's experience. Pediatr Neurol 2010; 43 155-158.

3. Coppola G, Grosso S, Franzoni E, et al. Rufinamide in refractory childhood epileptic encephalopathies other than Lennoxe-Gastaut syndrome. Eur J Neurol 2011; 18: $246-251$.

4. Vendrame M, Poduri A, Loddenkemper T, Kluger G, Coppola G, Kothare SV. Treatment of malignant migrating partial epilepsy of infancy with rufinamide: Report of five cases. Epileptic Disord 2011; 13: 18-21.

5. Kluger G, Kurlemann G, Haberlandt E, et al. Effectiveness and tolerability of rufinamide in children and adults with refractory epilepsy: First European experience. Epilepsy Behav 2009; 14: 491-495.

6. Grosso S, Coppola G, Dontin SD, et al. Efficacy and safety of rufinamide in children under four years of age with drug-resistant epilepsies. Eur J Paediatr Neurol 2014: 18; 641-645.

7. White HS, Franklin MR, Kupferberg HJ, Schmutz M, Stables JP, Wolf HH. The anticonvulsant profile of rufinamide (CGP 33101) in rodent seizure models. Epilepsia 2008; 49: 1213-1220.

8. Perucca E, Cloyd J, Critchley D, Fuseau E. Rufinamide: Clinical pharmacokinetics and concentration-response relationships in patients with epilepsy. Epilepsia 2008 49: 1123-1141.

9. Wirrell EC. Predicting pharmacoresistance in pediatric epilepsy. Epilepsia 2013; 54(Suppl 2): 19-22.

10. Epilepsy ILa. [Accessed 2nd July 2015]; Guidelines for Publications from League Commissions and Task Forces. 2014 Available at: http://www.ilae.org/visitors/ centre/guidelines.cfm.

11. Jaraba S, Santamarina E, Miró J, et al. Rufinamide in children and adults in routine clinical practice. Acta Neurol Scand 2017; 135: 122-128.

12. McMurray R, Striano P. Treatment of Adults with Lennox-Gastaut Syndrome: Further Analysis of Efficacy and Safety/Tolerability of Rufinamide. Neurol Ther 2016; 5: 35-43.

13. Glauser T, Kluger G, Sachdeo R, Krauss G, Perdomo C, Arroyo S. Rufinamide for generalized seizures associated with Lennox-Gastaut syndrome. Neurology 2008; 70: 1950-1958. 
14. Coppola G, Grosso S, Franzoni E, et al. Rufinamide in children and adults with Lennox-Gastaut syndrome: First Italian multicenter experience. Seizure 2010; 19: 587-591.

15. Kim SH, Eun SH, Kang HC, et al. Rufinamide as an adjuvant treatment in children with Lennox-Gastaut syndrome. Seizure 2012; 21: 288-291.

16. Lee EH, Yum MS, Ko TS. Effectiveness and tolerability of rufinamide in children and young adults with Lennox-Gastaut syndrome: A single center study in Korea. Clin Neurol Neurosurg 2013; 115: 926-929.
17. Mueller A, Boor R, Coppola G, et al. Low long-term efficacy and tolerability of add-on rufinamide in patients with Dravet syndrome. Epilepsy Behav 2011; 21: 282284.

18. Moavero R, Cusmai R, Specchio N, et al. Rufinamide efficacy and safety as adjunctive treatment in children with focal drug resistant epilepsy: the first Italian prospective study. Epilepsy Res 2012; 102: 94-99.

19. von Stulpnagel C, Leichsenring M, Muller A, Staudt M, Kluger G. Refractory focal epilepsy in a patient with methylmalonic aciduria: case report on positive and long lasting effect of rufinamide. Neuropediatrics 2011; 42: 71-73. 